\section{Trigeminusneuralgie und orofaziale} Schmerzen

Helga Peter

Marburg, Deutschland

\section{Definition}

Die Diagnose der verschiedenen Formen von Gesichtsneuralgien und -schmerzen erfolgt nach den Kriterien der International Headache Society, IHS (2003).

\section{Grundlagen}

Schmerzzustände im Bereich des Kopfes, wie z. B. orofaziale Schmerzen und die Trigeminusneuralgie, führen gehäuft zu Ein- und Durchschlafstörungen mit resultierender Tagesschläfrigkeit. Polysomnographisch wurden eine verminderte
Gesamtschlafzeit, eine erhöhte Anzahl von Wachphasen und Körperbewegungen und ein reduzierter Tiefschlafanteil bei Patienten mit orofazialen Schmerzen nachgewiesen. Die Trigeminusneuralgie wird entsprechend der Therapieempfehlungen der Deutschen Migräne- und Kopfschmerz-Gesellschaft (www.dmkg.de) behandelt. Kurzfristig können in Einzelfällen „Benzodiazepine“ den Schlaf von Patienten mit chronischen orofazialen Schmerzen verbessern, für die längerfristige Therapie kann Amitriptylin erfolgreich eingesetzt werden.

Siehe auch

- $>$ „Kopfschmerz“

- $>$ „Kopfschmerzen bei Schlafstörungen“ 